Collectanea Mathematica (electronic version): http://www.collectanea.ub.edu

Collect. Math. 61, 2 (2010), 131-149

(C) 2010 Universitat de Barcelona

\title{
Characterizations of space curves containing a planar subcurve
}

\author{
Silvio Greco ${ }^{1}$ \\ Dipartimento di Matematica-Politecnico di Torino, I-10129 Torino, Italy \\ E-mail: silvio.greco@polito.it \\ Giuseppe Paxia ${ }^{2}$ \\ Dipartimento di Matematica-Università di Catania, Viale A. Doria, 6 \\ Catania-I-95125, Italy \\ E-mail: paxia@dmi.unict.it
}

Received April 4, 2009. Revised September 7, 2009

\begin{abstract}
We introduce and study the space curves of " $h$-extremal type", that are curves of degree $d$ and arithmetic genus $g$ whose Rao function agrees, in a suitable interval depending on $d$ and $h$, with the one of the " $h$-extremal"curves introduced by Notari-Sabadini. Our study is motivated by the literature of the last years concerning curves with large cohomology and their relations with the Hilbert scheme.

Our main result is a Structure Theorem which gives some geometrical characterizations of such curves. The most intriguing is that if $d$ is sufficiently large with respect to $h$, a curve of h-extremal type contains a planar subcurve of degree $d-h$ and lies on a non integral quadric. As a consequence we can determine all possible Rao functions (for fixed $d, g, h$ ). We add several examples which show, in particular, that our result is the best possible for $h \leq 5$.
\end{abstract}

\section{Introduction}

The problem of classifying curves $C$ in the projective space $\mathbb{P}_{k}^{3}$ is very old and far from being completely solved. For this reason many authors, following different approaches, are still working on this problem obtaining new and interesting results.

\footnotetext{
${ }^{1}$ Supported by MIUR and GNSAGA-INDAM.

${ }^{2}$ Supported by PRA-Catania University.

Keywords: Extremal and subextremal curves; Rao functions; Hilbert schemes.

MSC2000: Primary: 14H50.
} 
A line of research is to attach "discrete invariants" to the curves, trying to characterize all curves in $\mathbb{P}_{k}^{3}$ which share the same invariants.

This has brought, for instance, at the use of Chow variety and Hilbert scheme, to parameterize all curves of given degree $d$ and (arithmetic) genus $g$.

Many authors indeed focus their attention on the Rao function of a curve $C$, i.e. $\rho_{C}(j):=h^{1}\left(\mathcal{I}_{C}(j)\right)=\operatorname{dim} H^{1}\left(\mathcal{I}_{C}(j)\right)$, where $\mathcal{I}_{C} \subseteq \mathcal{O}_{\mathbb{P}^{3}}$ denotes the ideal sheaf of $C$ and $H^{1}\left(\mathcal{I}_{C}(j)\right)$ are the cohomology groups.

In this context the first remarkable result is due to Martin-Deschamps and Perrin in [15] where they prove the existence of a function $\rho^{E}(j): \mathbb{Z} \rightarrow \mathbb{Z}$ which maximizes all the Rao functions $\rho_{C}(j)$ for curves $C \subseteq \mathbb{P}^{3}$ of degree $d$ and arithmetic genus $g$. The curves for which the maximum is achieved are called "extremal curves".

In this direction S. Nollet in [19] showed that excluding the extremal curves the remaining ones are such that their Rao function $\rho_{C}(j)$ is in turn maximized by a function $\rho^{S E}(j)$; the curves which attain such maximal value are called "subextremal curves".

But such a procedure cannot continue. After excluding extremal and subextremal curves, it is not possible to find a function $\rho(j)$ which maximizes the Rao function of the remaining curves.

For this reason Chiarli, Greco and Nagel in [5] introduced the notion of curves of "subextremal type", which are curves whose Rao function agrees with $\rho^{S E}(j)$, when $1 \leq j \leq d-3$.

Another interesting approach is the study of bounds on the Rao function of a curve $C$ done by R.M. Miró-Roig and S. Nollet in [18] where they find upper bounds to Rao function in terms of the degree, genus and minimal degree $s$ of a surface $S$ containing $C$.

In all the previous papers interesting geometrical properties of the curves are related to algebraic and cohomological properties. For instance all the curves mentioned above have the common feature to be contained in one (or more) quadrics, to contain a planar subcurve, to have a special form of the Hilbert function. In all these studies many different points of view are considered.

In this paper we introduce the curves, which we call of " $h$-extremal type", with the property that their Rao function agrees, in a suitable range, with the Rao function of the $h$-extremal ones considered by Notari and Sabadini in [20].

The aim of this work is to extend to any integer $h$ results similar to those obtained in $[15,5]$, for the cases $h=1$ and $h=2$. But in our general setting the techniques and methods used are not a mere generalization of those used previously.

This paper contains four sections. In Section 2 we recall some notation, results and tools which we will use freely in the rest of the paper.

In Section 3 we give our basic definition of $h$-extremal type curves and state the Structure Theorem which is the main theorem of the paper. Moreover we deduce from it a complete description of the Rao functions of such curves (Proposition 3.5). In addition we prove that any "compatible" Rao function actually occurs (Proposition 3.7).

In Section 4, after proving some preparatory results, which might be of independent interest, we prove our main theorem.

Finally in the last section we give many different examples which can enlighten the theory developed and discuss about possible further investigations. In particular 
we treat the problem of the sharpness for the bounds on the degree $d$ that we need for the validity of the Structure Theorem. It is interesting that for a given $h \geq 4$ the sharpness of our bound is equivalent to the existence of a curve $C^{\prime}$ with assigned properties, see Proposition 5.2.

For basic facts on the classification of curves in $\mathbb{P}^{3}$ one can look at [14].

\section{Notation, tools and known results}

We recall some basic facts and results that will be used in the sequel.

a) $K$ : algebraically closed field of characteristic zero.

b) For a closed subscheme $X \subseteq \mathbb{P}^{n}, h_{X}$ denotes the Hilbert function of $X$ and $\partial h_{X}$ denotes the first difference of $h_{X}$, i.e. $\partial h_{X}(j)=h_{X}(j)-h_{X}(j-1)$.

c) If $X \subseteq \mathbb{P}^{n}$ is a closed subscheme, then $\mathcal{I}_{X} \subseteq \mathcal{O}_{\mathbb{P}^{n}}$ denotes the ideal sheaf of $X$ and $I_{X} \subseteq K\left[X_{0}, \ldots, X_{n}\right]$ denotes the (saturated) homogeneous ideal of $X$.

d) A curve $C \subseteq \mathbb{P}^{3}$ is a pure 1-dimensional projective subscheme without 0-dimensional components; in particular $C$ is locally Cohen-Macaulay. Usually with $\Gamma$ we will denote a general plane section of $C$.

e) If $C$ is a curve, the function $\rho_{C}(j):=h^{1}\left(\mathcal{I}_{C}(j)\right)=\operatorname{dim}_{K} H^{1}\left(\mathcal{I}_{C}(j)\right)(j \in \mathbb{Z})$, is called the Rao function of $C$.

As we mentioned in the introduction it is very interesting to describe properties of curves in terms of their Rao functions.

First of all we recall the definition of h-extremal curve given by Notari and Sabadini $[20]$.

Definition 2.1 Let $d, g$ integers. A curve $C$ of degree $d$ and genus $g$ is $h$-extremal for some $h \leq \frac{d}{2}$ if

(1) $h \geq 1, d-h \geq 1$;

(2) $C$ contains a planar curve of degree $d-h$;

(3) $\rho_{C}=\rho^{h-E}$, where $\rho^{h-E}$ is defined as follows:

$$
\rho^{h-E}(j):= \begin{cases}0 & \text { if } \quad j \leq r \\
\left(\begin{array}{c}
d-h-1 \\
2
\end{array}\right)+\left(\begin{array}{c}
h-1 \\
2
\end{array}\right)-g+j & \text { if } \quad r+1 \leq j<h-1 \\
\left(\begin{array}{c}
d-h-1 \\
2
\end{array}\right)+\left(\begin{array}{c}
h \\
2
\end{array}\right)-g & \text { if } \quad h-1 \leq j \leq d-h-1 \\
\left(\begin{array}{c}
d-h \\
2
\end{array}\right)+\left(\begin{array}{l}
h \\
2
\end{array}\right)-g-j & \text { if } \quad d-h-1<j \leq s-1 \\
0 & \text { if } \quad j \geq s\end{cases}
$$

where $r:=g-\left(\begin{array}{c}h-1 \\ 2\end{array}\right)-\left(\begin{array}{c}d-h-1 \\ 2\end{array}\right), \quad s:=\left(\begin{array}{c}d-h \\ 2\end{array}\right)+\left(\begin{array}{c}h \\ 2\end{array}\right)-g$. 
In [20] it is proved that $\rho^{h-E}$ is a sharp bound for the Rao function of a curve containing a planar subcurve of degree $d-h$, the bound being attained by the h-extremal curves. It is also worth to observe that $h$-extremal curves are characterized in [20, Theorem 3.10] as those that can be obtained from an extremal curve by an elementary biliaison of height $h-1$ on a quadric.

Essentially we can say that for such curves the Rao functions are trapezium "shifted", as $h$ is varying.

We notice that (with the notation as in the Introduction) $\rho^{1-E}=\rho^{E}$ and $\rho^{2-E}=\rho^{S E}$.

Recall that a curve $C$ is "extremal" if $\rho_{C}=\rho^{E}$ and "subextremal" if $\rho_{C}=\rho^{S E}$.

For extremal curves, the following characterization follows from $[8,15]$ :

\section{Theorem 2.2}

Suppose $d \geq 5$. Then the following are equivalent:

(a) $C$ is extremal;

(b) $C$ contains a planar subcurve of degree $d-1$;

(c) $C$ is contained in two independent quadrics.

(d) $\partial h_{\Gamma}: 1,2,1,1, \ldots$

It is worth observing that there does not exist a result like Theorem 2.2 for subextremal curves. This is one of the motivations for the following definition given in [5]:

Definition 2.3 A non-degenerate curve $C \subseteq \mathbb{P}^{3}$ of degree $d$ and genus $g$ is called of subextremal type if $d \geq 5$ and $\rho_{C}(j)=\left(\begin{array}{c}d-3 \\ 2\end{array}\right)-g+1$, for $1 \leq j \leq d-3$. nience.

In [5] it is proved the following theorem, which we recall for the reader's conve-

\section{Theorem 2.4}

Let $C \subseteq \mathbb{P}^{3}$ be a non-degenerate curve of degree $d \geq 7$. Then the following conditions are equivalent:

(i) $C$ is of subextremal type;

(ii) $h^{0}\left(\mathcal{I}_{C}(2)\right)=1$ and $h^{0}\left(\mathcal{I}_{C}(3)\right)=5$ (that is, $I_{C}$ has one minimal generator in degree 2 and one in degree 3$)$;

(iii) $C$ is contained in a unique quadric and $\partial h_{\Gamma}: 1221 \ldots 10 \rightarrow$;

(iv) $C$ contains a planar subcurve of degree $d-2$ and the residual curve $C^{\prime}$ is a planar curve of degree 2.

It is important for the sequel to emphasize that for the validity of Theorems 2.2 and 2.4 the degree of the curve must be greater than a certain integer. For instance in [5, Remark 3.3] there are two examples of curves of degree $d=5$ and $d=6$ for which Theorem 2.4 is not true.

\section{Residual sequences}

The notion of residual sequence will play a very useful role in many technical tools that we will use in our proofs. 
Suppose that $C$ contains a planar subcurve. Let $D \subset C$ be a planar subcurve of largest degree $d-\delta \leq d$. Denote by $H$ the plane spanned by $D$ and let $\ell \in R$ be a linear form defining $H$. Let $C^{\prime}$ be the residue of $C$ with respect to $H$, namely $\mathcal{I}_{C^{\prime}}:=\mathcal{I}_{C}: \mathcal{I}_{H}$, and let $Z \subseteq H$ be the residue of $C \cap H$ with respect to $D$, namely $\mathcal{I}_{Z, H}:=\mathcal{I}_{C \cap H, H}: \mathcal{I}_{D, H}$. Let $g^{\prime}$ denote the arithmetic genus of $C^{\prime}$.

\section{Proposition 2.5}

With the above notation we have:

(i) $\mathcal{I}_{Z, H}(\delta-d)$ is isomorphic to $\mathcal{I}_{C \cap H, H}$, via the multiplication by an equation of $D$;

(ii) there exists an exact sequence (called the residual sequence with respect to $H$ ):

$$
0 \rightarrow \mathcal{I}_{C^{\prime}}(-1) \rightarrow \mathcal{I}_{C} \rightarrow \mathcal{I}_{Z, H}(\delta-d) \rightarrow 0
$$

where the first map is the multiplication by $\ell$;

(iii) $C^{\prime}$ is a curve of degree $\delta$;

(iv) $Z$ is either empty or zero-dimensional;

(v) $\operatorname{deg}(Z)=\left(\begin{array}{c}d-\delta-1 \\ 2\end{array}\right)-g+g^{\prime}+\delta-1$;

(vi) $Z$ is a subscheme of $C^{\prime} \cap H$;

(vii) If $C^{\prime}$ is aCM we have: $\operatorname{deg} Z=h^{1}\left(\mathcal{I}_{C}(j)\right)$ for $\delta-1 \leq j \leq d-\delta-1$.

Proof. For properties (i) to (vi) see [5, Proposition 2.3].

(vii). By (iii) and standard arguments we have $h^{2}\left(\mathcal{I}_{C^{\prime}}(j)\right)=0$ for all $j \geq \delta-2$.

Then from the residual sequence (2.2) twisted by $j$ for $\delta-1 \leq j \leq d-\delta-1$ we get

$$
h^{1}\left(\mathcal{I}_{C}(j)\right)=h^{1}\left(\mathcal{I}_{Z, H}(\delta-d+j)\right)=\operatorname{deg}(Z),
$$

the last equality because $\delta-d+j<0$.

For basic facts on the Rao functions the interested reader can consult the book of J.C. Migliore [17].

\section{Structure Theorem and consequences}

First of all we give our basic definition, which is quite natural in view of Definitions 2.1 and 2.3:

Definition 3.1 A non degenerate curve $C$ of degree $d \geq 2 h+1$ and arithmetic genus $g$ is of " $h$-extremal type" (shortly h-ET) if

$$
\rho_{C}(j)=a:=\left(\begin{array}{c}
d-h-1 \\
2
\end{array}\right)-g+\left(\begin{array}{l}
h \\
2
\end{array}\right), \text { for } \quad h-1 \leq j \leq d-h-1 .
$$

Remark 3.2 (1) According to this definition the curves of 1-ET are exactly the extremal curves studied in [15]. This follows easily from the main result of [19] (see also [8]).

(2) The curves of 2-ET are the curve of subextremal type studied in [5], see Definition 2.3.

(3) Obviously the $h$-extremal curves (Definition 2.1) are h-ET, but the converse is false, as we shall see later, see Remark 3.8. 
In our general setting the Structure Theorem becomes

\section{Theorem 3.3}

Let $h \geq 2$ and let $C \subseteq \mathbb{P}^{3}$ be a non degenerate curve of degree $d$ and genus $g$. Consider the following conditions:

(i) $C$ is of h-extremal type;

(ii)

$$
h^{0}\left(\mathcal{I}_{C}(j)\right)=\left\{\begin{array}{lll}
\left(\begin{array}{c}
j+1 \\
3
\end{array}\right) & \text { if } & 2 \leq j \leq h \\
\left(\begin{array}{c}
j+1 \\
3
\end{array}\right)+1 & \text { if } & j=h+1
\end{array} .\right.
$$

That is $I_{C}$ has one minimal generator in degree 2 and the first independent generator is in degree $h+1$;

(iii) $C$ is contained in a unique quadric and $\partial h_{\Gamma}: 1 \underbrace{22 \cdots 2}_{h} 1 \cdots 1 \quad 0 \rightarrow$;

(iv) $C$ contains a planar subcurve $D$ of degree $d-h$ and the residual curve $C^{\prime}$ is a planar curve of degree $h$.

Then the following statements hold:

(a) if $d \geq 2 h+3$ then (ii), (iii), (iv) are equivalent and imply (i);

(b) if moreover $d \geq\left(\begin{array}{l}h \\ 3\end{array}\right)+2 h+1$ then (i) implies (iv) (and the four conditions are equivalent).

The proof of the Structure Theorem will be done in the next section. Here we deduce some interesting consequences of it.

\section{Corollary 3.4}

Let $C \subseteq \mathbb{P}^{3}$ be a curve of degree $d \geq 2 h+3$ with $h \geq 2$. Assume one of the following:

a) $C$ satisfies condition (iv) of Theorem 3.3.

b) $C$ is $h-E T$ and $d \geq\left(\begin{array}{l}h \\ 3\end{array}\right)+2 h+1$

Then $C$ is contained in a unique quadric $Q$ which is either the union of two distinct planes or a double plane.

Proof. Using the implications (i) $\Longrightarrow$ (iv) $\Longrightarrow$ (ii) of Theorem 3.3 we see that $C$ is contained in a unique quadric $Q$. Moreover by (ii) $C$ is also contained in a surface $F$ of degree $h+1$ with $Q \nsubseteq F$. Since $d>2 \operatorname{deg} F, Q$ and $F$ must have a common component by Bézout. The conclusion follows.

It is worth noticing that the most interesting and general situations occur when the curve $C$ is contained in a quadric $Q=2 H$, as we will see in the sequel.

At this point the reader can ask how the Rao function for curves of $h$-extremal type, satisfying the Structure Theorem, looks like. For this we state the following

\section{Proposition 3.5}

Let $h \geq 2$ and let $C \subseteq \mathbb{P}^{3}$ be a $h$-ET curve of genus $g$ and degree $d$ satisfying conditions (ii) to (iv) of Theorem 3.3 (e.g. assume $d \geq \max \left\{2 h+3,\left(\begin{array}{l}h \\ 3\end{array}\right)+2 h+1\right\}$ ). Let

$$
0 \rightarrow \mathcal{I}_{C^{\prime}}(-1) \rightarrow \mathcal{I}_{C} \rightarrow \mathcal{I}_{Z, H}(h-d) \rightarrow 0,
$$


be the residual sequence arising from (iv), and set

$$
a:=\left(\begin{array}{c}
d-h-1 \\
2
\end{array}\right)-g+\left(\begin{array}{l}
h \\
2
\end{array}\right)
$$

Then $a=\operatorname{deg} Z$ (Proposition 2.5). Moreover we have:

(i) the Rao function $\rho_{C}$ is symmetric. Precisely:

$$
\rho_{C}(j)=\rho_{C}(d-2-j) \text { for all } j \in \mathbb{Z} .
$$

(ii) $\rho_{C}$ is completely determined by $h_{Z}$. Precisely:

$$
\rho_{C}(j)=a-h_{Z}(j-d+h) \text { for } j \geq d-h
$$

(the rest by symmetry). Moreover:

$$
\partial \rho_{C}(j)=-\partial h_{Z}(j-d+h) \text { for } j \geq d-h .
$$

(iii) $C$ is h-extremal (Definition 2.1) if and only if $Z$ is collinear.

(iv) If the quadric containing $C$ is reduced then $C$ is $h$-extremal.

(v) For all $j, \rho_{C}(j) \leq a$.

(vi) $g \leq\left(\begin{array}{c}d-h-1 \\ 2\end{array}\right)+\left(\begin{array}{l}h \\ 2\end{array}\right)$. Equality holds if and only if $C$ is aCM.

Proof. (i) By Corollary 3.4 we know that there are two possibilities: either the unique quadric $Q$ containing $C$ is a double plane and then the symmetry follows by [11, Corollary 6.2], or it is reduced; in this case the symmetry of $\rho_{C}(j)$ is guaranteed by [7, Corollary 2.4].

(ii) From the residual sequence we get the exact sequence, for $j \geq d-h$ :

$$
\begin{aligned}
0=H^{1}\left(\mathcal{I}_{C^{\prime}}(-1+j)\right) & \longrightarrow H^{1}\left(\mathcal{I}_{C}(j)\right) \longrightarrow H^{1}\left(\mathcal{I}_{Z, H}(h-d+j)\right) \\
& \longrightarrow H^{2}\left(\mathcal{I}_{C^{\prime}}(-1+j)\right)=0
\end{aligned}
$$

whence

$$
\rho_{C}(j)=h^{1}\left(\mathcal{I}_{Z}(h-d+j)\right)=\operatorname{deg} Z-h_{Z}(h-d+j)=a-h_{Z}(h-d+j) .
$$

(iii) By Theorem 3.3(iv) $C$ contains a planar subcurve of degree $d-h$. The conclusion is obvious by (ii) and Definition 2.1.

(iv) We know by Proposition 2.5(vi) that $Z \subseteq H \cap H^{\prime}$ (where $H \cup H^{\prime}$ is the quadric containing $C$ ) and hence it is collinear. So by (iii) $C$ is $h$-extremal.

(v) By (ii) $\rho_{C}(j)$ is non-increasing for $j \geq h-1$, whence $\rho_{C}(j) \leq a$ for $j \geq h-1$. The conclusion follows from (i).

(vi) Obvious from (v).

\section{Remark 3.6}

As a consequence of the previous Proposition 3.5 we can describe the Rao functions of $h$-extremal type curves. We know that in general $\left.h^{1} \mathcal{I}_{Z, H}(t)\right)=\operatorname{deg} Z-h_{Z}(t)$. Hence the decreasing of $\left.h^{1} \mathcal{I}_{Z, H}(t)\right)$ depends on the increasing of $h_{Z}(t)$. We know that 
$Z \subset C^{\prime} \cap H$, where $H$ is the plane of the subcurve of degree $d-h$. Let $k$ be the minimal degree of a curve containing $Z$; obviously if $Z \neq \emptyset$ is $1 \leq k \leq h=\operatorname{deg} C^{\prime}$.

We know that $\partial h_{Z}$ must be of the form:

$$
\partial h_{Z}: \quad 1 \quad 2 \quad \cdots \quad \underbrace{k \cdots k}_{a_{1}} \underbrace{(k-1) \cdots(k-1)}_{a_{2}} \cdots
$$

where $a_{1}, a_{2}, \ldots, a_{k-1}$ are integers greater than or equal to 0 . Just $a_{1}$ must be $>0$.

To give an idea we can summarize the values for the Rao function $\rho_{C}(j)=h^{1}\left(\mathcal{I}_{C}(j)\right)$ as in the following table

\begin{tabular}{||c|c|c|c|}
\hline$j$ & $t=d-h+j$ & $h^{1}\left(\mathcal{I}_{Z}(t)\right)$ & $h^{1}\left(\mathcal{I}_{C}(j)\right)$ \\
\hline \hline$d-h$ & 0 & $\operatorname{deg} Z-1$ & $a-1$ \\
\hline$d-h+1$ & 1 & $\operatorname{deg} Z-3$ & $a-3$ \\
\hline$\vdots$ & $\vdots$ & $\vdots$ & $\vdots$ \\
\hline$d-h+(k-1)$ & $k-1$ & $\operatorname{deg} Z-\left(\begin{array}{c}k+1 \\
2\end{array}\right)$ & $a-\left(\begin{array}{c}k+1 \\
2\end{array}\right)$ \\
\hline$d-h+k$ & $k$ & $\operatorname{deg} Z-\left(\begin{array}{c}k+1 \\
2\end{array}\right)-k$ & $a-\left(\begin{array}{c}k+1 \\
2\end{array}\right)-k$ \\
\hline$\vdots$ & $\vdots$ & $\vdots$ & $\vdots$
\end{tabular}$\|$

where $a=\left(\begin{array}{c}d-h-1 \\ 2\end{array}\right)-g+\left(\begin{array}{l}h \\ 2\end{array}\right)$, cf. Definition 3.1.

Now we want to show that all "compatible" Rao functions actually occur. Precisely:

\section{Proposition 3.7}

Fix integers $h \geq 2, d \geq 2 h+3, g \leq\left(\begin{array}{c}d-h-1 \\ 2\end{array}\right)+\left(\begin{array}{l}h \\ 2\end{array}\right)$ and set $a:=\left(\begin{array}{c}d-h-1 \\ 2\end{array}\right)-g+\left(\begin{array}{l}h \\ 2\end{array}\right)$. Then we have:

(i) let $Z \subseteq \mathbb{P}^{2}$ be a reduced zero-dimensional scheme of degree $a$, with $h^{0}\left(\mathcal{I}_{Z}(h) \neq 0\right.$. Then there exists a h-ET curve $C$ of degree $d$ and genus $g$ such that

$$
\rho_{C}(j)=\rho_{C}(d-2-j) \quad \text { for all } j \in \mathbb{Z}
$$

and

$$
\rho_{C}(j)=a-h_{Z}(j-d+h) \text { for } j \geq d-h .
$$

(ii) Let $f: \mathbb{Z} \rightarrow \mathbb{Z}$ be a function such that $f(0)=1$ and $\partial f$ has the form (3.1) for some $k \leq h$ and $\sum_{j \geq 0} \partial f(j)=a$.

Then there exists a h-ET curve $C$ of degree $d$ and genus $g$ such that $\rho_{C}$ satisfies (3.2) and

$$
\rho_{C}(j)=a-f(j-d+h) \quad \text { for } \quad j \geq d-h .
$$

Proof. (i) Identify $\mathbb{P}^{2}$ with a plane $H \subseteq \mathbb{P}^{3}$. Let $C^{\prime}, D \subseteq H$ be curves, of degrees $h$ and $d-h$ respectively, such that $Z \subseteq C^{\prime} \subseteq D$. Since $Z$ is locally a complete intersection there exists a curve $C \subseteq 2 H$ containing $D$ and with residual sequence

$$
0 \rightarrow \mathcal{I}_{C^{\prime}}(-1) \rightarrow \mathcal{I}_{C} \rightarrow \mathcal{I}_{Z, H}(h-d) \rightarrow 0
$$


(see [11] or [4, Corollary 4.5]). In particular $C$ satisfies condition (iv) of Theorem 3.3. The conclusion follows then from Theorem 3.3 and Proposition 3.5.

(ii) It is well known that there exists a reduced zero-dimensional scheme $Z \subseteq \mathbb{P}^{2}$ such that $h_{Z}=f$ (see e.g. [9, Theorem 4.1] or [13, Theorem 4]). The conclusion follows from (a).

Remark 3.8 Let $h, d, g, a$ be as in Proposition 3.7. Then:

(i) if $a>2$ there are h-ET curves which are not h-extremal: just apply Proposition 3.5(iii) and Proposition 3.7(i) with a non-collinear $Z$.

(ii) Let $t:=\max \left\{j \in \mathbb{Z} \mid\left(\begin{array}{c}j+2 \\ 2\end{array}\right) \leq a\right\}$ and define $f$ as follows:

$$
f(j)=\left\{\begin{array}{lll}
\left(\begin{array}{c}
j+2 \\
2
\end{array}\right) & \text { if } & j<t \\
a-\left(\begin{array}{c}
t+2 \\
2
\end{array}\right) & \text { if } & j=t \\
a & \text { if } & j>t
\end{array}\right.
$$

Then f satisfies the assumptions of Proposition 3.7(b) and the corresponding h-ET curves have the smallest Rao function in the set of all h-ET curves of degree $d$ and genus $g$ (details to the reader).

Remark 3.9 In $[4,7]$ one can find a complete description of the homogeneous ideal and of the Hartshorne-Rao module of a curve lying on a non-integral quadric. Clearly this applies to h-ET curves satisfying (iv) of Theorem 3.3.

\section{Proof of the Structure Theorem}

To prove the theorem we need some preparation. We start by recalling a useful lemma:

\section{Lemma 4.1}

Let $C \subseteq \mathbb{P}^{3}$ be a non degenerate curve and $\Gamma$ be a general plane section. Suppose that for some $t \in \mathbb{Z}$ we have $h^{1}\left(\mathcal{I}_{\Gamma}(t)\right)=0$ and $h^{1}\left(\mathcal{I}_{C}(t)\right) \neq 0$. Then $h^{1}\left(\mathcal{I}_{C}(t)\right)>$ $h^{1}\left(\mathcal{I}_{C}(t+1)\right)$. In other words the Rao function $\rho_{C}(j)$ is strictly decreasing until is zero, starting from the value where the Hilbert function of $\Gamma$ reaches the multiplicity.

Proof. See for instance [1, Lemma 4.6].

\section{Proposition 4.2}

Let $C \subseteq \mathbb{P}^{3}$ be a non degenerate curve of "h-extremal type", with $h \geq 2$ and $d \geq 2 h+3$. Then:

(a) $C$ contains a planar curve $D$ of degree $d-\delta$ for some $\delta \in\{2, \cdots, h\}$.

(b) Let $C^{\prime}$ be the residual curve of $C$ with respect to the plane $H$ of $D$. Then

(b1) $H^{1}\left(\mathcal{I}_{C^{\prime}}(j)\right)=H^{2}\left(\mathcal{I}_{C^{\prime}}(j)\right)=0$ for $j \geq h-2$. 
(b2) the arithmetic genus of $C^{\prime}$ is:

$$
g^{\prime}=\left(\begin{array}{c}
d-h-1 \\
2
\end{array}\right)-\left(\begin{array}{c}
d-\delta-1 \\
2
\end{array}\right)+1-\delta+\left(\begin{array}{l}
h \\
2
\end{array}\right)
$$

Proof. (a) We start by proving that, under our hypothesis,

$$
h^{1}\left(\mathcal{I}_{\Gamma}(d-h-2)\right) \neq 0
$$

For this we have to distinguish two cases:

I) $\left(\begin{array}{c}d-h-1 \\ 2\end{array}\right)-g+\left(\begin{array}{l}h \\ 2\end{array}\right)>0$.

II) $g=\left(\begin{array}{c}d-h-1 \\ 2\end{array}\right)+\left(\begin{array}{l}h \\ 2\end{array}\right)$.

Case I). By assumption it is

$$
h^{1}\left(\mathcal{I}_{C}(d-h-2)\right)=h^{1}\left(\mathcal{I}_{C}(d-h-1)\right) \neq 0
$$

and applying the Lemma 4.1 , it must be $h^{1}\left(\mathcal{I}_{\Gamma}(d-h-2)\right) \neq 0$.

Case II). We assume that $h^{1}\left(\mathcal{I}_{\Gamma}(d-h-2)\right)=0$ and we seek for a contradiction. We know that

$$
\begin{aligned}
g & =1-\chi\left(\mathcal{O}_{C}\right) \\
& =h^{1}\left(\mathcal{O}_{C}\right)-h^{0}\left(\mathcal{O}_{C}\right)+1 \\
& \leq h^{1}\left(\mathcal{O}_{C}\right) \\
& =h^{2}\left(\mathcal{I}_{C}\right) .
\end{aligned}
$$

By [5, Theorem 3.2] (proof of $i) \Rightarrow i i)$ ) or [8, Lemma 1], it follows $h^{2}\left(\mathcal{I}_{C}\right) \leq$ $\sum_{j>0} h^{1}\left(\mathcal{I}_{\Gamma}\right)(j)$ and then, under our hypotheses,

$$
\left(\begin{array}{c}
d-h-1 \\
2
\end{array}\right)+\left(\begin{array}{l}
h \\
2
\end{array}\right) \leq \sum_{j=1}^{d-h-3} h^{1}\left(\mathcal{I}_{\Gamma}\right)(j) .
$$

We want to find a good upper bound for right hand side of (4.2), compatibly with our hypotheses.

For convenience we put $g(j):=h^{1}\left(\mathcal{I}_{\Gamma}(j)\right)$. Since $g(j)=d-h_{\Gamma}(j)$ it follows that it is strictly decreasing until it is zero. The maximum value of $\sum_{j=1}^{d-h-3} g(j)$ is reached when we have the minimum decreasing at each step, compatibly with the condition $g(d-h-2)=0$. It is easy to see that a decreasing by 1 at each step is not possible; we have to allow a decreasing by 2 for a certain number of steps, the minimum possible, and then a decreasing by 1 .

Since the curve $C$ is not planar it is $g(1)=d-3$, according to [10, Theorem 2.1]. Intersecting two suitable lines of slope respectively -2 and -1 one gets the best situation:

$$
g(j+1)= \begin{cases}g(j)-2 & \text { for } 1 \leq j \leq h \\ g(j)-1 & \text { for } h+1 \leq j \leq d-h-3 .\end{cases}
$$

From (4.3) we obtain

$$
\begin{aligned}
g(1)+\cdots+g(h+1) & \leq(d-3)+(d-5)+\cdots+[d-1-2(h+1)] \\
& =(h+1)(d-h-3)
\end{aligned}
$$




$$
\begin{aligned}
g(h+2)+\cdots+g(d-h-3) & \leq(d-2 h-4)+\cdots+2+1 \\
& =\frac{(d-2 h-4)(d-2 h-3)}{2} .
\end{aligned}
$$

By substituting (4.4) and (4.5) in (4.2) we get

$$
\left(\begin{array}{c}
d-h-1 \\
2
\end{array}\right)+\left(\begin{array}{l}
h \\
2
\end{array}\right) \leq(h+1)(d-h-3)+\frac{(d-2 h-4)(d-2 h-3)}{2} .
$$

The previous inequality implies $d \leq 2 h+2$ contradicting our assumption; so $h^{1}\left(\mathcal{I}_{\Gamma}(d-h-2)\right) \neq 0$ holds in any case.

It follows at once $h_{\Gamma}(d-h-2)<d$.

We put $\partial h_{\Gamma}(i):=a_{i}$, for $0 \leq i \leq d-h-1$. First of all we get $\sum_{i=0}^{d-h-2} a_{i} \leq d-1$, but $a_{d-h-1}>0$.

Now letting $\delta:=\sum_{i=0}^{d-h-2}\left(a_{i}-1\right)$ it follows at once $\delta \leq d-1-(d-h-2+1)=h$.

We prove now that at least two values among the $a_{i}$ are necessarily equal to 1 . Namely if $a_{d-h-2}=1$ it follows that $a_{d-h-1}=1$, since the function $\partial h_{\Gamma}$ is not increasing. If $a_{d-h-2} \geq 2$, from $a_{0}+a_{1}+\cdots+a_{d-h-2} \leq d-1$ it would follow $1+2(d-h-2) \leq d-1$ and then $d \leq 2 h+2$; this contradicts the hypothesis. Then it follows necessarily $a_{d-h-2}=a_{d-h-1}=1$.

Notice that $d-\delta=1+\max \left\{i \mid a_{i} \neq 0\right\}$. Since $a_{d-h-2}=a_{d-h-1}=\cdots=a_{d-\delta-1}=1$, it follows from [12, Theorem 2.9] or [6, Theorem 4.1] that $\Gamma$ has a subscheme of degree $d-\delta$ spanning a line, and contains no collinear subscheme of larger degree. Furthermore, $d-\delta \geq(d-h-1)+1=d-h \geq \frac{d+3}{2}$, the latter inequality coming from the fact that $d \geq 2 h+3$. It then follows from [3, Corollary 4.4.] that $C$ contains a planar curve $D$ of degree $d-\delta$. Obviously $\delta \neq 0$ since otherwise $C$ would be planar. Furthermore $\delta \neq 1$, since otherwise $C$ would be extremal by Theorem 2.2, contradicting the assumption $h \geq 2$. Thus we have $2 \leq \delta \leq h$ as claimed.

(b1) Let $\Gamma^{\prime}$ be a general plane section of $C^{\prime}$. Then $H^{1}\left(\mathcal{I}_{\Gamma^{\prime}}(\delta-1)\right)=0$, whence

$$
H^{1}\left(\mathcal{I}_{\Gamma^{\prime}}(h-1)\right)=0 .
$$

From (4.6) it follows:

$$
H^{2}\left(\mathcal{I}_{C^{\prime}}(j)\right)=0 \text { for } j \geq h-2 .
$$

Consider now the residual sequence

$$
0 \longrightarrow \mathcal{I}_{C^{\prime}}(-1) \longrightarrow \mathcal{I}_{C} \longrightarrow \mathcal{I}_{Z, H}(\delta-d) \longrightarrow 0
$$

and let $j \in\{h-1, h\}$. Then $\delta-d+j<0$, whence $H^{0}\left(\mathcal{I}_{Z, H}(\delta-d+j)\right)=0$ and $H^{1}\left(\mathcal{I}_{Z, H}(\delta-d+j)\right)=\operatorname{deg}(Z)$. Now from (4.7) and the cohomology sequence of (4.8) we get an exact sequence for $j \in\{h-1, h\}$ :

$$
0 \longrightarrow H^{1}\left(\mathcal{I}_{C^{\prime}}(j-1)\right) \longrightarrow H^{1}\left(\mathcal{I}_{C}(j) \longrightarrow H^{1}\left(\mathcal{I}_{Z, H}(\delta-d+j)\right) \longrightarrow 0 .\right.
$$

Since $h \leq d-h-1$ we have $h^{1}\left(\mathcal{I}_{C}(h-1)\right)=h^{1}\left(I_{C}(h)\right)$, hence (4.9) implies $h^{1}\left(\mathcal{I}_{C^{\prime}}(h-2)\right)=h^{1}\left(I_{C^{\prime}}(h-1)\right)$. The conclusion follow from (4.6) and Lemma 4.1. 
(b2) From (4.9) with $j=h-1$ and (b1) we get $h^{1}\left(\mathcal{I}_{C}(h-1)\right)=\operatorname{deg}(Z)$. Hence by Proposition 2.5(v) we obtain:

$$
\left(\begin{array}{c}
d-h-1 \\
2
\end{array}\right)-g+\left(\begin{array}{l}
h \\
2
\end{array}\right)=\left(\begin{array}{c}
d-\delta-1 \\
2
\end{array}\right)-g+g^{\prime}+\delta-1
$$

whence the conclusion.

The following result is probably well-known, but we include it for lack of a ready reference.

\section{Proposition 4.3}

Let $X \subseteq \mathbb{P}^{3}$ be a curve of degree $n$. Then $H^{0}\left(\mathcal{I}_{X}(n)\right) \neq 0$.

Proof. Let $Y \subseteq \mathbb{P}^{2}$ be a general projection of $X$. Then $\operatorname{dim}(Y)>0$ and with a suitable choice of homogeneous coordinates we have: $I_{X} \subseteq K\left[X_{0}, X_{1}, X_{2}, X_{3}\right]$ and $I_{Y}:=I_{X} \cap K\left[X_{0}, X_{1}, X_{2}\right]$. Then $K\left[X_{0}, X_{1}, X_{2}\right] / I_{Y}$ is a graded subring of $K\left[X_{0}, X_{1}, X_{2}, X_{3}\right] / I_{X}$ and this implies $h_{Y}(j) \leq h_{X}(j)$ for every $j$. In particular we have $p_{Y}(j) \leq p_{X}(j)$ for $j \gg 0$. Then $\operatorname{dim}(Y) \leq \operatorname{dim}(X)$ whence $\operatorname{dim}(Y)=1$. Since $X$ has no zero-dimensional components and the projection is general it is easy to see that $Y$ has no zero-dimensional components, whence $I_{Y}$ is principal. Moreover by comparing the leading terms of the Hilbert polynomials we see that $\operatorname{deg}(Y) \leq \operatorname{deg}(X)$. Hence $I_{Y}=(F)$, where $F$ is a homogeneous polynomial of degree $\leq n$. Now $I_{Y}$ is a subset of $I_{X}$ and hence $F$, viewed as an element of $K\left[X_{0}, X_{1}, X_{2}, X_{3}\right]$, is a polynomial of degree $\leq n$ belonging to $I_{Y}$.

Finally we can prove the Structure Theorem 3.3.

Proof. We prove (a). If $h=2$ the statement is true by Theorem 2.4. Then we let $h>2$ and we proceed by induction, assuming that (a) holds for any $k$ such that $2 \leq k<h$. We shall use the following pattern of implications:

$$
\text { (ii) } \Longrightarrow \text { (iii) } \Longrightarrow \text { (iv) } \Longrightarrow(\text { ii }) \vee(\text { i) }
$$

(ii) $\Longrightarrow$ (iii). We start by considering the exact sequence

$$
0 \rightarrow H^{0}\left(\mathcal{I}_{C}(1)\right) \rightarrow H^{0}\left(\mathcal{I}_{C}(2)\right) \rightarrow H^{0}\left(\mathcal{I}_{\Gamma}(2)\right) \rightarrow \cdots
$$

From this it follows $h^{0}\left(\mathcal{I}_{\Gamma}(2)\right) \geq 1$. If $h^{0}\left(\mathcal{I}_{\Gamma}(2)\right) \geq 2$ then $\partial h_{\Gamma}: 121 \rightarrow \cdots$ and $C$ is extremal by Theorem 2.2, contradicting the assumption $h \geq 2$. Then we must have:

$$
\partial h_{\Gamma}: 1 \underbrace{22 \cdots 2}_{k} y \cdots
$$

with $k>1$ and $y \leq 1$. We show first that $k \geq h$.

Assume $2 \leq k<h$. Then by hypothesis we have

$$
h^{0}\left(\mathcal{I}_{C}(k+1)\right)=\left(\begin{array}{c}
k+2 \\
3
\end{array}\right) .
$$


Since $d \geq 2 k+3$, we have $y=1$, whence (iii) is satisfied with $h$ replaced by $k$. Then by induction also (ii) is satisfied, with $h$ replaced by $k$. It follows

$$
h^{0}\left(\mathcal{I}_{C}(k+1)\right)=\left(\begin{array}{c}
k+2 \\
3
\end{array}\right)+1,
$$

a contradiction. Then $k \geq h$ and so we must have:

$$
\partial h_{\Gamma}: 1 \underbrace{22 \cdots 2}_{h} x \cdots .
$$

It remains to show that $x=1$. Now from the exact sequence

$$
0 \rightarrow \mathcal{I}_{C}(h) \rightarrow \mathcal{I}_{C}(h+1) \rightarrow \mathcal{I}_{\Gamma}(h+1) \rightarrow 0
$$

one deduces

$$
h^{0}\left(\mathcal{I}_{\Gamma}(h+1)\right) \geq h^{0}\left(\mathcal{I}_{C}(h+1)\right)-h^{0}\left(\mathcal{I}_{C}(h)\right)=\left(\begin{array}{c}
h+2 \\
3
\end{array}\right)+1-\left(\begin{array}{c}
h-2+3 \\
3
\end{array}\right) .
$$

Then $h^{0}\left(\mathcal{I}_{\Gamma}(h+1) \geq 1+\left(\begin{array}{c}h+1 \\ 2\end{array}\right)\right.$. From this it follows $h_{\Gamma}(h+1) \leq 2 h+2$ and then $1+2 h+x \leq 2 h+2$. Since $x \neq 0$, otherwise $d=2 h+1$, it must be $x=1$.

(iii) $\Longrightarrow$ (iv). By [3, Corollary 4.4], since $d-h \geq \frac{d+3}{2} \quad C$ must contain a unique planar curve $D$ of degree $d-h$. The residual sequence with respect to $H$, the plane containing $\mathrm{D}$, can be written

$$
0 \rightarrow \mathcal{I}_{C^{\prime}}(-1) \stackrel{\cdot H}{\longrightarrow} \mathcal{I}_{C} \rightarrow \mathcal{I}_{C \cap H, H} \rightarrow 0
$$

where $C^{\prime}$ is a curve of degree $h$ and the first map is the multiplication by a linear form $\ell$ defining $H$. Taking cohomology one has

$$
0 \longrightarrow H^{0}\left(\mathcal{I}_{C^{\prime}}(1)\right) \longrightarrow H^{0}\left(\mathcal{I}_{C}(2)\right) \longrightarrow H^{0}\left(\mathcal{I}_{C \cap H, H}(2)\right) \longrightarrow .
$$

Since $d-h>2$ it follows that $H^{0}\left(\mathcal{I}_{C \cap H, H}(2)\right)=0$ and then $h^{0}\left(\mathcal{I}_{C^{\prime}}(1)\right)=h^{0}\left(\mathcal{I}_{C}(2)\right)=$ 1. This implies that $C^{\prime}$ is a planar curve of degree $h$.

(iv) $\Longrightarrow$ (ii). We start again by considering the residual sequence

$$
0 \longrightarrow \mathcal{I}_{C^{\prime}}(-1) \longrightarrow \mathcal{I}_{C} \longrightarrow \mathcal{I}_{C \cap H, H} \longrightarrow 0
$$

and let $2 \leq j \leq h+1$. Twisting by $j$ and taking cohomology we obtain an exact sequence:

$$
0 \longrightarrow H^{0}\left(\mathcal{I}_{C^{\prime}}(j-1)\right) \longrightarrow H^{0}\left(\mathcal{I}_{C}(j)\right) \longrightarrow H^{0}\left(\mathcal{I}_{C \cap H, H}(j)\right) \longrightarrow 0 .
$$

But $H^{0}\left(\mathcal{I}_{C \cap H, H}(j)\right)=0$ for $j<d-h$, in particular for $2 \leq j \leq h+1$. Then we have:

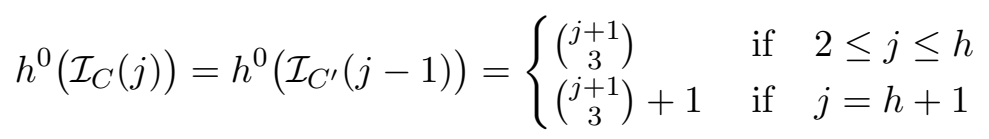

as we wanted to prove. 
(iv) $\Longrightarrow$ (i). We have the residual sequence

$$
0 \longrightarrow \mathcal{I}_{C^{\prime}}(-1) \longrightarrow \mathcal{I}_{C} \longrightarrow \mathcal{I}_{Z, H}(h-d) \longrightarrow 0
$$

where $H$ is the plane containing the planar curve $D$ of degree $d-h$ and $Z \subseteq H$ is the residual scheme of $C \cap H$ with respect to $D$. By Proposition 2.5(v) and (vii) we have, for $h-1 \leq j \leq d-h-1$ :

$$
\begin{aligned}
h^{1}\left(\mathcal{I}_{C}(j)\right) & =h^{1}\left(\mathcal{I}_{Z, H}(h-d+j)\right)=\operatorname{deg}(Z) \\
& =\left(\begin{array}{c}
d-h-1 \\
d-2
\end{array}\right)-g+g^{\prime}+h-1 \\
& =\left(\begin{array}{c}
d-h-1 \\
2
\end{array}\right)-g+\frac{1}{2}(h-1)(h-2)+h-1 \\
& =\left(\begin{array}{c}
d-h-1 \\
2
\end{array}\right)-g+\left(\begin{array}{c}
h \\
2
\end{array}\right) .
\end{aligned}
$$

From this it follows that $C$ is h-ET.

This concludes the proof of (a). The proof of (b) consists of just one implication, namely:

(i) $\Longrightarrow$ (iv) [with the extra assumption $d \geq\left(\begin{array}{l}h \\ 3\end{array}\right)+2 h+1$ ].

By Proposition $4.2 C$ contains a planar subcurve of degree $d-\delta(2 \leq \delta \leq h)$. Moreover the residual curve $C^{\prime}$ (of degree $\delta$ ) satisfies $h^{1}\left(\mathcal{I}_{C^{\prime}}(h-2)\right)=h^{2}\left(\mathcal{I}_{C^{\prime}}(\bar{h}-2)\right)=0$ and has arithmetic genus

$$
g^{\prime}=\left(\begin{array}{c}
d-h-1 \\
2
\end{array}\right)-\left(\begin{array}{c}
d-\delta-1 \\
2
\end{array}\right)+1-\delta+\left(\begin{array}{l}
h \\
2
\end{array}\right) .
$$

If $\delta=h$ it follows $g^{\prime}=\frac{1}{2}(h-1)(h-2)$ and then $C^{\prime}$ is a planar curve and we are done.

So it is sufficient to show that the inequality $\delta<h$ leads to a contradiction. Consider the exact sequence

$$
0 \longrightarrow \mathcal{I}_{C^{\prime}} \longrightarrow \mathcal{O}_{\mathbb{P}^{3}} \longrightarrow \mathcal{O}_{C^{\prime}} \longrightarrow 0 .
$$

Taking cohomology after twisting by $h-2$ and using Riemann-Roch we get:

$$
0=h^{0}\left(\mathcal{I}_{C^{\prime}}(h-2)\right)-\left(\begin{array}{c}
h+1 \\
3
\end{array}\right)+(h-2) \delta+1-g^{\prime}
$$

$\left(\right.$ remember that $\left.h^{1}\left(\mathcal{I}_{C^{\prime}}(h-2)\right)=h^{2}\left(\mathcal{I}_{C^{\prime}}(h-2)\right)=0\right)$. It follows:

$$
g^{\prime}=h^{0}\left(\mathcal{I}_{C^{\prime}}(h-2)\right)+(h-2) \delta+1-\left(\begin{array}{c}
h+1 \\
3
\end{array}\right) .
$$

Assume now $\delta=h-1$. Combining (4.10) and (4.12) we get, being $h^{0}\left(\mathcal{I}_{C^{\prime}}(h-2)\right) \geq$ 0 :

$$
-d+1+\frac{h^{2}-h}{2}+2 \geq(h-2)(h-1)+1-\left(\begin{array}{c}
h+1 \\
3
\end{array}\right)
$$

whence

$$
d \leq \frac{h^{3}-3 h^{2}+14 h}{6}=\left(\begin{array}{c}
h \\
3
\end{array}\right)+2 h
$$


a contradiction.

Suppose now $\delta \leq h-2$. Since by Proposition $4.3 C^{\prime}$ is contained in a surface of degree $\delta$ we have easily:

$$
h^{0}\left(\mathcal{I}_{C^{\prime}}(h-2)\right) \geq\left(\begin{array}{c}
h-2-\delta+3 \\
3
\end{array}\right)
$$

Hence by (4.12) we get

$$
\begin{aligned}
g^{\prime} & \geq(h-2) \delta+1-\left(\begin{array}{c}
h+1 \\
3
\end{array}\right)+\left(\begin{array}{c}
h+1-\delta \\
3
\end{array}\right) \\
& =(h-2) \delta+1+\frac{1}{6}[(h+1-\delta)(h-\delta)(h-1-\delta)-h(h+1)(h-1)] \\
& =(h-2) \delta+1+\frac{1}{6}\left[-\delta^{3}+3 h \delta^{2}-\left(3 h^{2}-1\right) \delta\right] .
\end{aligned}
$$

Using (4.10) we get, after an elementary computation:

$$
d \leq \frac{1}{6}[\underbrace{-\delta^{2}+(2 h+3) \delta-\left(h^{2}-9 h-4\right)}_{f(\delta)}+\frac{h^{3}-3 h^{2}+2 h}{h-\delta}] .
$$

It is easy to check that for $2 \leq \delta \leq h-2$ the maximum of $f(\delta)$ is achieved for $\delta=h-2$ and is $12 h-6$. Moreover $\frac{h^{3}-3 h^{2}+2 h}{h-\delta} \leq \frac{h^{3}-3 h^{2}+2 h}{2}=3\left(\begin{array}{l}h \\ 3\end{array}\right)$. It follows

$$
d \leq \frac{1}{6}\left[12 h-6+3\left(\begin{array}{l}
h \\
3
\end{array}\right)\right]=2 h-1+\frac{1}{2}\left(\begin{array}{l}
h \\
3
\end{array}\right)<\left(\begin{array}{l}
h \\
3
\end{array}\right)+2 h,
$$

again a contradiction.

\section{Examples and final remarks}

In this final section we want to discuss several examples which can enlighten the theory developed in the previous sections.

In particular we want to discuss the sharpness of the bounds for $d$ used for the validity of the Structure Theorem 3.3.

Recall that there are two bounds:

$$
d \geq 2 h+3
$$

(used for the equivalence of (ii),(iii),(iv) and the implication (iv) $\Longrightarrow($ i)), and

$$
d \geq\left(\begin{array}{l}
h \\
3
\end{array}\right)+2 h+1
$$

used for the implication (i) $\Longrightarrow$ (iv).

Clearly $2 h+3 \geq\left(\begin{array}{l}h \\ 3\end{array}\right)+2 h+1$ only for $h \leq 3$.

We use freely the notation of the previous section.

Our first example shows that the bound (5.1) is sharp for every $h$. 
EXAmPle 5.1 Assume $h \geq 2$ and let $C:=Q \cap F$ be a complete intersection where $Q$ is a smooth quadric and $F$ is a general surface of degree $h+1$. Then $d=2 h+2$, $g=h^{2}$ and $a=0$; see Proposition 3.7 for definition of $a$. It follows that $C$ satisfies conditions (i) (ii) and (iii) of Theorem 3.3. Moreover $C$ is smooth irreducible and hence it doesn't satisfy condition (iv) of the same Theorem. Then conditions (ii) (iii) (iv) are not equivalent without assuming (5.1).

In particular for $h=3$ the Structure Theorem holds if $d \geq 9$, but is false for $d=8$.

Note that for $h=2$ we get another counterexample for $d=6$, which completes the ones given in [5, Remark 3.3].

Now we want to discuss the bound (5.2). We show first that the sharpness of this bound for a given $h \geq 4$ is equivalent to the existence of a certain curve $C^{\prime}$ of degree $h-1$.

\section{Proposition 5.2}

Let $d=\left(\begin{array}{l}h \\ 3\end{array}\right)+2 h$ and $h \geq 4$. Then the following conditions are equivalent:

a) There exists a curve $C$ of $h$-extremal type and degree $d$, with $h^{0}\left(\mathcal{I}_{C}(2)\right)=0$.

b) There exists a curve $C^{\prime}$ satisfying the following conditions:

b1) $h^{1}\left(\mathcal{I}_{C^{\prime}}(j)\right)=0$ for $j \geq h-2$;

b2) $\operatorname{deg}\left(C^{\prime}\right)=h-1$;

b3) the arithmetic genus of $C^{\prime}$ is $g^{\prime}=-\left(\begin{array}{c}h-1 \\ 3\end{array}\right)-h+2$;

b4) $h^{0}\left(\mathcal{I}_{C^{\prime}}(h-2)\right)=0$.

c) There exists $C$ as in a) with the property: $h^{1}\left(\mathcal{I}_{C}(j)\right)=h-1$ for $h-1 \leq j \leq d-h-1$.

The degree $d$ considered in this Proposition is exactly one less than the degree $\frac{h^{3}-3 h^{2}+14 h+6}{6}$ from which the the implication (i) $\Longrightarrow$ (iv) of the Structure Theorem is true.

Proof. a) $\Longrightarrow$ b) Let $C^{\prime}$ be the curve of degree $\delta$ used in the proof of Theorem 3.3, (i) $\Longrightarrow$ (iv). We claim that $C^{\prime}$, under our assumption on $d$, satisfies b).

We use freely notation and calculations of the above mentioned proof. We see immediately that b1) holds.

Now we prove b2). Indeed if $\delta=h, C$ satisfies (iv), so it lies on a quadric by Corollary 3.4, contradicting a). On the other hand if $\delta \leq h-2$ it follows by (4.14) that $d<\left(\begin{array}{l}h \\ 3\end{array}\right)+2 h$, again a contradiction. So b2) holds.

Putting $\delta=h-1$ in (4.10) we get b3).

Finally substituting the values of $d, g^{\prime}$ and $\delta=h-1$ in (4.13) we get b4).

b) $\Longrightarrow$ c) Let $H$ be a plane in general position and let $D$ be a curve of degree $d-(h-1)$ lying on $H$, such that $D \cap C^{\prime}=\emptyset$. Let $C:=C^{\prime} \cup D$. Then adopting the usual notation, we have $Z=C^{\prime} \cap H$.

By the residual sequence with respect to $H$ we have for $h-1 \leq j \leq d-h-1$

$$
0 \rightarrow \mathcal{I}_{C^{\prime}}(j-1) \rightarrow \mathcal{I}_{C}(j) \rightarrow \mathcal{I}_{Z, H}(\delta-d+j) \rightarrow 0 .
$$


Taking into account all our hypotheses, by a technique already used many times in this paper it follows that $h^{1}\left(\mathcal{I}_{C}(j)=\operatorname{deg}(Z)\right.$ for $h-1 \leq j \leq d-h-1$. Now from Proposition 2.5(v) we have, since $\delta=h-1$ :

$$
\operatorname{deg}(Z)=\left(\begin{array}{c}
d-h \\
2
\end{array}\right)-g+g^{\prime}+h-2
$$

where $g:=p_{a}(C)$. By a straightforward computation, substituting the values of $d$ and $g^{\prime}$ in the previous formula, it follows by definition that $C$ is h-ET.

Since by hypothesis $h^{0}\left(\mathcal{I}_{C^{\prime}}(h-2)\right)=0$ the residual sequence easily implies $h^{0}\left(\mathcal{I}_{C}(2)\right)=0$.

c) $\Longrightarrow a$ ) Obvious.

Remark 5.3 We want to observe explicitly that the conditions listed in b) of Proposition 5.2 are not independent. More precisely if b2) holds, then any two among the conditions b1), b3, b4) implies the third. This follows easily from (4.12) and RiemannRoch, remembering that $H^{2}\left(\mathcal{I}_{C^{\prime}}(h-2)\right)=0$. We leave the details to the reader.

Now we use Proposition 5.2 to show that (5.2) is sharp for $h=4$ and $h=5$. First we recall some properties of the curves of degree 2 .

Remark 5.4 For any $g \leq 0$ there is a curve $X$ of degree 2 and arithmetic genus $g$. Any such curve $X$ is extremal and hence $\rho_{X}$ is described by (2.1), with $h=1$. In particular $h^{1}\left(\mathcal{I}_{X}(j)\right)=0$ for $j \geq-g$.

If $g \leq-2 X$ is necessarily a double line and the quadrics containing it are exactly the quadrics which are singular at each point of $X$.

(for details see e.g. [16, Proposition 0.6(ii)]).

Example 5.5 Let $h=4$. Then (5.2) is $d \geq 13$. Let $C^{\prime}:=E \cup L$ with $L$ a line and $E$ a double line of genus -2 such that $E \cap L=\emptyset$. Then $\operatorname{deg}\left(C^{\prime}\right)=3, h^{0}\left(\mathcal{I}_{C^{\prime}}(2)\right)=0$ and $p_{a}\left(C^{\prime}\right)=-3$. Then by Remark $5.3 C^{\prime}$ satisfies b) of Proposition 5.2. Then there is a 4 -ET curve $C$ of degree 12 not lying on a quadric. This means that (5.2) is sharp for $h=4$.

Example 5.6 Let $h=5$. Then (5.2) is $d \geq 21$. Let $C^{\prime}=A \cup B$ where $A$ and $B$ are disjoint double lines of genus -3 , hence the arithmetic genus of $C^{\prime}$ is $g^{\prime}=-7$. We want to prove that $H^{1}\left(\mathcal{I}_{C^{\prime}}(3)\right)=0$.

Since the lines are disjoint, the Mayer-Vietoris exact sequence is

$$
0 \rightarrow \mathcal{I}_{C^{\prime}} \rightarrow \mathcal{I}_{A} \oplus \mathcal{I}_{B} \rightarrow \mathcal{I}_{A \cap B}=\mathcal{O}_{\mathbb{P}^{3}} \rightarrow 0 .
$$

Since $h^{0}\left(\mathcal{I}_{X}(3)\right)=\left(I_{X}\right)_{3}$ we have the exact sequence

$$
\left.0 \rightarrow\left(I_{C^{\prime}}\right)_{3} \rightarrow\left(I_{A}\right)_{3} \oplus\left(I_{B}\right)_{3} \rightarrow R_{3} \rightarrow H^{1}\left(\mathcal{I}_{C^{\prime}}(3)\right) \rightarrow H^{1}\left(\mathcal{I}_{A}\right)(3)\right) \oplus H^{1}\left(\mathcal{I}_{B}(3)\right) \rightarrow 0
$$

where $R=k[x, y, z, t]$.

From Remark 5.4 we have: $H^{1}\left(\mathcal{I}_{A}(3)\right)=H^{1}\left(\mathcal{I}_{B}((3))=0\right.$, hence it is sufficient to prove that $f:\left(I_{A}\right)_{3} \oplus\left(I_{B}\right)_{3} \rightarrow R_{3}$ is surjective. 
Remembering how the Mayer-Vietoris sequence is defined we have $\operatorname{Im}(f)=\left(I_{A}\right)_{3}+$ $\left(I_{B}\right)_{3}$, so we have to show that $\left(I_{A}\right)_{3}+\left(I_{B}\right)_{3}=R_{3}$. We may assume that $A$ and $B$ are supported respectively by the lines $\left\{\begin{array}{l}x=0 \\ y=0\end{array}\right.$ and $\left\{\begin{array}{l}z=0 \\ t=0\end{array}\right.$. Then by Remark 5.4 $I_{A}$ contains the monomials: $x^{2}, x y, y^{2}$ and $I_{B}$ contains the monomials: $z^{2}, z t, t^{2}$. It follows easily $\left(I_{A}\right)_{3}+\left(I_{B}\right)_{3}$ contains all the monomials of degree 3 , whence our claim.

Then by Remark $5.3 C^{\prime}$ satisfies b) of Proposition 5.2, and this shows that there is a 5 -ET curve $C$ of degree 20 not contained in a quadric.

Remark 5.7 The above Examples 5.5 and 5.6 show that (5.2) is sharp for $h=4$ and $h=5$. We don't know whether this is true for $h \geq 6$.

For example the sharpness for $h=6$ is equivalent to the existence of a curve $C^{\prime}$ of degree 5 and genus $g^{\prime}=-14$, satisfying $H^{0}\left(\mathcal{I}_{C^{\prime}}(4)\right)=0$ or, equivalently, $H^{1}\left(\mathcal{I}_{C^{\prime}}(4)\right)=0$.

Our last example shows that for the validity of the implication (i) $\Longrightarrow(v)$ of the Structure Theorem at least a quadratic bound is necessary.

EXAMPLE 5.8 Let $h \geq 4$ and set $d:=\frac{h^{2}+h+2}{2}$.

We construct an h-ET curve of degree $d$ which is not contained in a quadric, contradicting the condition (ii) (hence (iii) and (iv)) of the Structure Theorem.

Let $C$ be the disjoint union of two curves $C^{\prime}$ and $D$, where $C^{\prime}$ is a $(0, h-1)$ curve on a smooth quadric and $D$ is a planar curve of degree $d-(h-1)$ not meeting $C^{\prime}$.

By construction $C$ doesn't lie on a quadric. However by an argument as in the proof of Proposition 5.2, b) $\Longrightarrow \mathrm{c}$ ), one can show that $C$ is h-ET (we leave the details to the reader).

Note that in particular if $h=4$ we have a 4-ET curve of degree 11 not lying on a quadric.

We end by pointing out some questions and possible developments.

Our first question is related to the curves $C^{\prime}$ arising in Proposition 5.2(b).

Remark 5.9 As we have seen the existence of the curves $C^{\prime}$ satisfying (b) of Proposition 5.2 is equivalent to the sharpness of the bound (5.2). But there is something else.

For a curve $C \subseteq \mathbb{P}^{3}$ set $s(C):=\min \left\{j \mid H^{0}\left(\mathcal{I}_{C}(j)\right) \neq 0\right\}$. Let $C^{\prime}$ be as in Proposition 5.2, b). Then $s\left(C^{\prime}\right)=\operatorname{deg}\left(C^{\prime}\right)$, which is the maximum allowed by Proposition 4.3.

Observe also that $C^{\prime}$ has maximal rank.

It would be interesting to classify the curves with this property, independently from their relevance with respect to Theorem 3.3.

It seems that the first problem is to figure out a bound for the Rao function and the arithmetic genus for a curve $C^{\prime}$ such that $s\left(C^{\prime}\right)=\operatorname{deg}\left(C^{\prime}\right)$ (with or without the maximal rank assumption). Note that the results in [18] always assume $d \geq 2 s$, hence they cannot be applied directly.

Another problem is to understand the structure of the family of the h-ET curves of given degree and genus and its relations with the Hilbert scheme. 
Remark 5.10 The case $h=1$ (that is extremal curves) is completely described in [16] where it is shown, in particular, that for a given pair $d, g$ they form an irreducible component of the Hilbert scheme.

In [5] the family of 2-ET curves is studied for $d \geq 7$, and relations with the Hilbert scheme are established when $g<0$.

We feel that for $h \geq 3$ similar results should hold if the bound (5.2) is satisfied, but that an intricate structure might occur for lower $d$. This is of course related to the previous remark when $d=\left(\begin{array}{l}h \\ 3\end{array}\right)+2 h$, and looks even more intricate for lower $d$ 's.

\section{References}

1. M. Brodmann and U. Nagel, Bounding cohomological Hilbert functions by hyperplane sections, J. Algebra 174 (1995), 323-348.

2. N. Chiarli, S. Greco, and U. Nagel, On the genus and Hartshorne-Rao module of projective curves, Math. Z. 229 (1998), 695-724.

3. N. Chiarli, S. Greco, and U. Nagel, When does a projective curve contain a planar subcurve?, J. Pure Appl. Algebra 164 (2001), 345-364.

4. N. Chiarli, S. Greco, and U. Nagel, Normal form for space curves in a double plane, J. Pure Appl. Algebra 190 (2004), 45-57.

5. N. Chiarli, S. Greco, and U. Nagel, Families of space curves with large cohomology, J. Algebra 307 (2007), 704-726.

6. E.D. Davis, Complete intersections of codimension 2 in $\mathbb{P}^{r}$ : the Bezout-Jacobi-Segre theorem revisitated, Rend. Sem. Mat. Univ. Politec. di Torino 43 (1985), 333-353.

7. R. Di Gennaro and U. Nagel, The equations of space curves on a quadric, Collect. Math. 58 (2007), $119-130$.

8. Ph. Ellia, On the cohomology of projective space curves, Boll. Un. Mat. Ital. A (7) 9 (1995), 593-607.

9. A. Geramita, P. Maroscia, and J. Roberts, The Hilbert function of a reduced k-algebra, J. London Math. Soc. (2) 28 (1983), 443-452.

10. R. Hartshorne, The genus of space curves, Ann. Univ. Ferrara-Sez. VII (N.S.) 40 (1994), 207-223, 1996.

11. R. Hartshorne and E. Schlesinger, Curves in the double plane, (Special issue in honor of Robin Hartshorne), Comm. Algebra 28 (2000), 5655-5676.

12. R. Maggioni and A. Ragusa, Connections between Hilbert function and geometric properties for a finite set of points in $P^{2}$, Matematiche (Catania) 39 (1984), 153-170.

13. R. Maggioni and A. Ragusa, The Hilbert function of generic plane sections of curves of $\mathbb{P}^{3}$, Invent. Math. 91 (1988), 253-258.

14. M. Martin-Deschamps and D. Perrin, Sur la classification des courbes gauches, Astérisque 184-185 (1990), 208.

15. M. Martin-Deschamps and D. Perrin, Sur les bornes du module de Rao, C. R. Acad. Sci. Paris Sér. I Math. 317 (1993), 1159-1162.

16. M. Martin-Deschamps and D. Perrin, Le schéma de Hilbert des courbes gauches localement de Cohen-Macaulay n'est (presque) jamais réduit, Ann. Sci. École Norm. Sup. (4) 29 (1996), 757-785.

17. J.C. Migliore, Introduction to Liaison Theory and Deficiency Modules, Progress in Mathematics 165, Birkhäuser Boston, Inc., Boston, MA, 1998.

18. R.M. Miró-Roig and S. Nollet, Bounds on the Rao function, J. Pure Appl. Algebra 152 (1999), $253-266$.

19. S. Nollet, Subextremal curves, Manuscripta Math. 94 (1997), 303-317.

20. R. Notari and I. Sabadini, On the cohomology of a space curve containing a plane curve, Comm. Algebra 29 (2001), 4795-4810. 